PHYSICAL REVIEW SPECIAL TOPICS - PHYSICS EDUCATION RESEARCH 3, 029901(E) (2007)

\title{
Publisher's Note: Strongly and weakly directed approaches to teaching multiple representation use in physics [Phys. Rev. ST Phys. Educ. Res. 3, 010108 (2007)]
}

Patrick B. Kohl, David Rosengrant, and Noah D. Finkelstein

(Received 26 July 2007; published 3 August 2007)

DOI: 10.1103/PhysRevSTPER.3.029901

PACS number(s): 01.40.Fk, 99.10.Fg

This paper was published online on 12 June 2007 with an incorrect byline address for the second author in the author list. David Rosengrant's byline address should read as "Kennesaw State University, Kennesaw, Georgia 30144-5591". The address has been corrected as of 27 July 2007. 